\title{
FORMULATION AND EVALUATION OF ALLOPURINOL LOADED CHITOSAN NANOPARTICLES
}

\author{
GURPREET KANDAV*, D. C. BHATT, DEEPAK KUMAR JINDAL
}

Department of Pharmaceutical Sciences, Guru Jambheshwar University of Science and Technology, Hisar, Haryana 125001, India Email: gurpreetk11.1990@gmail.com

Received: 08 Jan 2019, Revised and Accepted: 05 Mar 2019

\section{ABSTRACT}

Objective: The objective of the present investigation was to fabricate and characterize allopurinol loaded chitosan nanoparticles (A-CNPs) for sustained release of drug.

Methods: The allopurinol loaded chitosan nanoparticles were successfully prepared by employing the ionotropic gelation method. Further, particle size (PS), polydispersity index (PDI), zeta potential (ZP), Differential Scanning Calorimetry (DSC), entrapment efficiency (EE), Transmission Electron Microscopy (TEM), in vitro drug release, X-Ray Diffraction (XRD) and Fourier transform infrared (FTIR) were used for evaluating formulated A-CNPs

Results: A-CNPs was successfully prepared and the particle size, polydispersity index, ZP and entrapment efficiency were found to be $375.3 \pm 10.1$ $\mathrm{nm}, 0.362 \pm 0.01$ and $32.5 \pm 2.7 \mathrm{mV}$ and $52.56 \pm 0.10 \%$ respectively. In vitro release profile of A-CNPs showed sustained release and Higuchi model was found to be best fit for drug release kinetics. FTIR study depicted no chemical interaction between pure drug allopurinol (AL) and other excipients.

Conclusion: The sustained release formulation of allopurinol was successfully prepared using HMW chitosan and evaluated for different parameters.

Keywords: Allopurinol, Sustained release, Chitosan, Nanoparticles

(C) 2019 The Authors. Published by Innovare Academic Sciences Pvt Ltd. This is an open-access article under the CC BY license (http://creativecommons.org/licenses/by/4.0/) DOI: http://dx.doi.org/10.22159/ijap.2019v11i3.31932

\section{INTRODUCTION}

Allopurinol (4 hydroxy pyrazolo (3, 4-d) pyrimidine), is a hypoxanthine isomer. Allopurinol (AL) acts by competitively inhibiting the enzyme xanthine oxidase (XOA), which is responsible for catalyzing the oxidation of normal purines to uric acid (UA) [1]. Allopurinol is a UA lowering therapeutic agent mainly used for the treatment of hyperuricemia and its related complications such as UA nephrolithiasis, hyperuricosuric, gout, urate nephropathy etc. [2, 3]. Allopurinol is very slightly soluble in water, possesses a short half-life of $1-2 \mathrm{~h}$ and its oral dose is $100-300 \mathrm{mg}$ (once a day). The poor solubility and shorter half-life leads to lesser bioavailability of drug via the oral route and this strongly indicates the urge for the development of sustained release formulation of allopurinol for enhancing bioavailability [4]. Colloidal drug carriers such as nanoparticles have attained a potential place in the controlled drug delivery systems. Nanoparticles are solid polymeric colloidal particles, in which the therapeutic agent is either encapsulated, entrapped or adsorbed onto the surface. They are prepared from natural (chitosan, alginate, gelatin etc.) or artificial (polylactides, polymethacrylate etc.) polymers and their size ranges from 10-1000 $\mathrm{nm}$ [5-7]. Various factors have to be considered while selecting a polymer for developing polymeric nanoparticles such as desired nano-particle size and drug release profile, biocompatibility, toxicity, biodegradability, drug inherent property and surface characteristics. Chitosan is a naturally occurring polysaccharide, obtained after deacetylation of chitin and it is studied extensively for the development of polymeric nanoparticles. Chitosan has various traits like chemical versatility, biocompatibility, biodegradability, non-toxic, cost effectiveness etc, which makes it an appropriate choice as a drug delivery carrier [8]. In the present investigation, sustained release allopurinol loaded chitosan nanoparticles (A-CNPs) were prepared by ionotropic gelation method where chitosan was crosslinked to tripolyphosphate (TPP) molecules that will protect the drug from harsh gastrointestinal environment and improves drug bioavailability and solubility by nanonization. The formulated A-CNPs was also evaluated for different parameters such as PS, entrapment efficiency, in vitro drug release, FTIR study etc.

\section{MATERIALS AND METHODS}

\section{Materials}

Allopurinol (AL) was received as a gift sample from Indoco Remedies Ltd. Maharashtra, India. High molecular weight (HMW) chitosan (CS) and sodium tripolyphosphate (TPP) was obtained from SRL Pvt Ltd, Mumbai, India. Acetic acid glacial (99.8\%) was sourced from Thomas Baker Chemicals, India. All other reagents used in this research work were of analytical grade.

Preparation of Allopurinol loaded chitosan nanoparticles (ACNPs)

A-CNPs were fabricated by employing the modified ionotropic geltation method [9]. CS solution $(0.2 \% \mathrm{w} / \mathrm{v})$ was prepared by dissolving HMW CS in $2 \%$ glacial acetic acid under continuous stirring and $\mathrm{pH}$ adjustment to 5.6 was made by adding sodium hydroxide solution. Subsequently, 100 $\mathrm{mg}$ of allopurinol was added to the freshly prepared CS solution and stirred for $30 \mathrm{~min}$. Further, an aqueous solution of crosslinker TPP was prepared in deionized water for obtaining a TPP: chitosan ratio of 1:3. TPP solution was then injected dropwise to the above solution and kept on $1 \mathrm{~h}$ stirring (1000 rpm) to allow electrostatic interactions among two oppositely charged moieties for the formation of A-CNPs. The dispersion was then, centrifuged at $15000 \mathrm{rpm}\left(4^{\circ} \mathrm{C}, 30 \mathrm{~min}\right)$ for isolating nanoparticles as pellet and the supernatant was collected for further analysis. The pellet was washed, re-dispersed in deionized water $(10 \mathrm{ml})$ and again centrifuged at $15000 \mathrm{rpm}\left(4^{\circ} \mathrm{C}, 30 \mathrm{~min}\right)$ to obtain nanoparticles in the pellet form. The pellet was redispersed in $10 \mathrm{ml}$ deionized water and this dispersion was later utilized for evaluating various parameters. The A-CNPs dispersion was finally freeze-dried by employing lyophilizer.

\section{Entrapment efficiency}

The supernatant collected after centrifugation of A-CNPs dispersion was analyzed in triplicate for estimating the amount of free drug. The absorbance of the supernatant sample was measured by using UV spectrophotometer at $\lambda$ max $250 \mathrm{~nm}$ against suitable blank. Entrapment efficiency was calculated by using the equation mentioned below [10]:

Drug content estimation $=\frac{\text { Total AL content }- \text { free AL content }}{\text { Total AL content }} * 100$

\section{PS, PDI and ZP}

Suitable dilution of A-CNPs dispersion (10 times) was prepared for estimating the particle size (PS), polydispersity index (PDI) and zeta potential (ZP) in triplicate on Zetasizer (Malvern Instruments, U. K.) by using dynamic light scattering technique. The scattering was recorded at $90^{\circ}$ angle and $25^{\circ} \mathrm{C}$ temperature $[11,12]$. 


\section{Transmission electron microscopy}

TEM was performed for examining the surface morphology and PS of formulated A-CNPs by using High-resolution transmission electron microscope (TECNAI, at $200 \mathrm{kV}$ ). Sample for TEM analysis was prepared by suitably diluting the A-CNPs with HPLC grade water and further sonicated for ensuring particle deaggregation. A drop of dispersion was put on a copper grid coated with carbon film (300 mesh) and stained (2\% phosphotungstic acid, $\mathrm{pH}$ 7) before recording TEM images $[13,14]$.

\section{In vitro drug release}

The release profile of AL from A-CNPs was evaluated through dialysis technique on a USP paddle (Type 2) dissolution apparatus. The dissolution medium $(500 \mathrm{ml})$ consisted of phosphate buffer $(\mathrm{pH}$ $7.4,37^{\circ} \mathrm{C} \pm 0.5^{\circ} \mathrm{C}$ ) with paddle rotating speed of $100 \mathrm{rpm}$. A-CNPs (equivalent to $4 \mathrm{mg} \mathrm{AL}$, redispersed in $3 \mathrm{ml}$ phosphate buffer) was introduced inside the dialysis bag which was previously soaked for 1 $\mathrm{h}$ in distilled water before the experiment and the bag was safely closed from both ends. The dialysis bag was then immersed in dissolution medium and $5 \mathrm{ml}$ sample aliquots were withdrawn at regular intervals of time from dissolution media and were replaced with an equal amount of fresh buffer solution for maintaining sink conditions. The aliquots were then analyzed (triplicate) spectrophotometrically at $\lambda \max =250 \mathrm{~nm}$ for determining percent drug release. The data procured from the in vitro study of A-CNPs was further evaluated for different drug release kinetic models (Higuchi, Korsemeyer Peppas, Hixson-Crowell, zero order, and first order) $[15,16]$

\section{Fourier-transform infrared spectroscopy}

FTIR spectrum of pure AL, HMW CS, dummy chitosan nanoparticles (CNPs) i. e without drug and lyophilized allopurinol loaded chitosan nanoparticles (A-CNPs) were observed on FTIR spectrophotometer (4000-400 $\left.\mathrm{cm}^{-1}\right)$ by using $\mathrm{KBr}$ method. FTIR spectroscopy was carried out for investigating any kind of chemical interactions among pure AL and other excipients in A-CNPs formulation [17].

\section{X-ray diffraction}

X-ray diffractograms of pure AL and lyophilized A-CNPs were procured on X-ray diffractometer (Rigaku MiniFlex II) for examining the physical state of pure drug (AL) and its interaction with other carriers in the formulation. The source of X-ray was CopperK $\alpha$ $\left(\lambda=1.5405{ }^{\circ} \mathrm{A}\right)$ monochromatic radiation, operated at $30 \mathrm{kV}$ and $15 \mathrm{~mA}$. The samples were scanned between 2 theta range of $10^{\circ}-80$ ${ }^{\circ}$, with an increment in a step size of $0.02^{\circ}$ and time of $2 \mathrm{~s}$ [18].

\section{Differential scanning calorimetry}

DSC thermograms of AL, lyophilized A-CNPs, CNPs and physical mixture of AL and HMW chitosan (P-AC) were acquired on DSC equipment (TA Instruments, Q-10, USA), which was operated in the temperature range of $25{ }^{\circ}$ to $400{ }^{\circ} \mathrm{C}$ under nitrogen inert environment with heat flowing at $10{ }^{\circ} \mathrm{C} / \mathrm{min}$. DSC analysis was performed for investigating the polymorphic changes in drug state in formulation [19].

\section{RESULTS AND DISCUSSION}

\section{Entrapment efficiency (EE)}

The percent EE of A-CNPs was determined by employing the abovementioned method and it was found to be $52.56 \pm 0.10 \%$ (mean $\pm S D, n=3$ ).

\section{PS, PDI and ZP}

The mean PS (hydrodynamic diameter), PDI and ZP of A-CNPs were observed to be $375.3 \pm 10.1 \mathrm{~nm}, 0.362 \pm 0.01$ and $32.5 \pm 2.7 \mathrm{mV}$ respectively $(n=3)$. The formulated A-CNPs was found to be in nano range and low value of polydispersity index signifies the formulation to be stable and monodisperse in nature. Further, high positive ZP of A-CNPs suggest greater electrostatic repulsion between particles in dispersion and thus making it more stable by preventing aggregation [13].

\section{Transmission electron microscopy}

The TEM micrograph of A-CNPs has been displayed in fig. 1, which indicates that the particles were present as discrete units and having a spherical shape. The PS of A-CNPs as obtained by TEM technique was in the range of $230-290 \mathrm{~nm}$, which was lesser than that procured by zetasizer. This may have occurred due to the dehydration of A-CNPs while preparing its sample for TEM analysis [9].

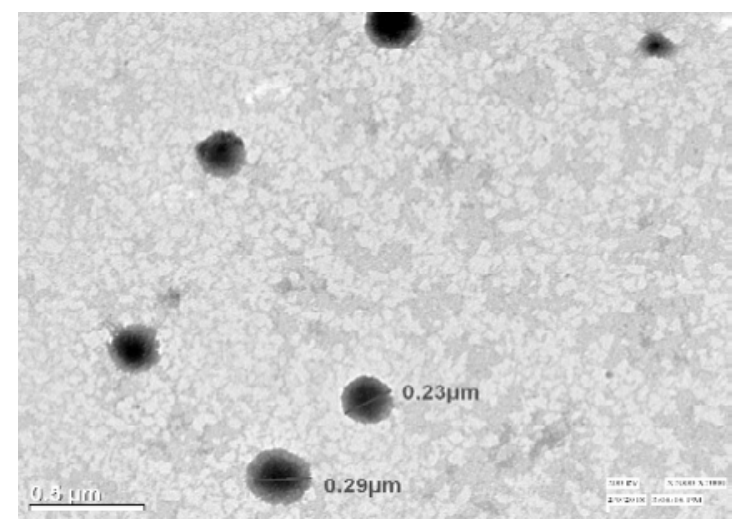

Fig. 1: TEM micrograph of A-CNPs

\section{In vitro drug release}

Fig. 2, depicts the sustained release pattern of allopurinol from A-CNPs and it showed \% cumulative release of $51.58 \pm 0.86 \%(\mathrm{n}=3)$ in $6 \mathrm{~h}$. The data acquired from in vitro study of A-CNPs was further analysed with different kinetic models (Higuchi, Hixson-Crowell, KorsemeyerPeppas, first order and zero model). The maximum linearity with correlation coefficient $\left(\mathrm{R}^{2}\right)$ of 0.9916 was obtained for Higuchi model, signifying drug release from A-CNPs was mainly controlled by diffusion process and the value of release component (n) was found to be 0.59 which lies in the range of 0.45 and 0.89 , depicting that the drug release follows non Fickian transport mechanism [15].

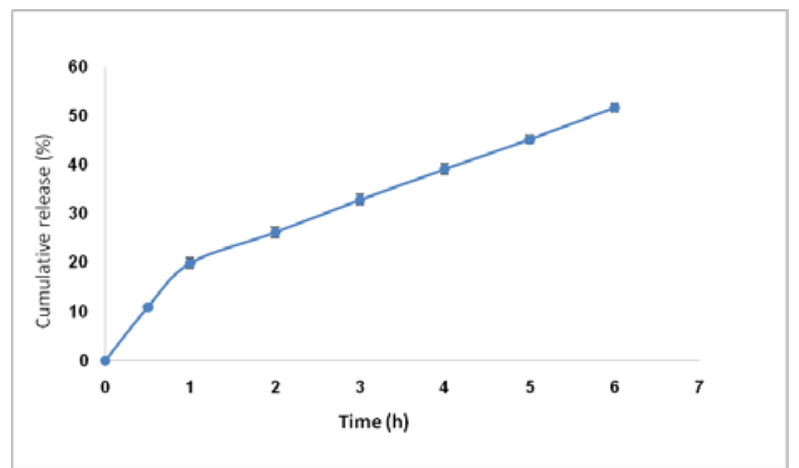

Fig. 2: In vitro drug release profile of A-CNPs, mean $\pm S D, n=3$

\section{Fourier-transform infrared spectroscopy}

FTIR spectrum of AL, HMW CS, CNPs and lyophilized A-CNPs are shown in fig. 3. The principal peaks at 3360, 3043, 1705, 1764 and $1593 \mathrm{~cm}^{-1}$ were obtained in the spectrum of AL [20]. The spectra of HMW chitosan showed a broad band at $3416 \mathrm{~cm}^{-1}(\mathrm{OH})$ and sharp peaks at $2956 \mathrm{~cm}^{-1}(\mathrm{CH})$ and $1654 \mathrm{~cm}^{-1}(\mathrm{NH})$. In the CNPs, the 1654 $\mathrm{cm}^{-1}$ peak of $\mathrm{NH}$ bending disappears and a new peak at 1643 $\mathrm{cm}^{-1}$ appears, which can be ascribed to the linkage between tripolyphosphate and ammonium group of TPP and chitosan, respectively. Also, a new peak at $1224 \mathrm{~cm}^{-1}$ (PO stretching) appeared in the CNPs which was not present in the spectra of pure HMW chitosan [21]. All the principal peaks of pure drug AL were 
present in FTIR spectra of lyophilized A-CNPs, thus indicating no chemical interaction between pure $\mathrm{AL}$ and other excipients.

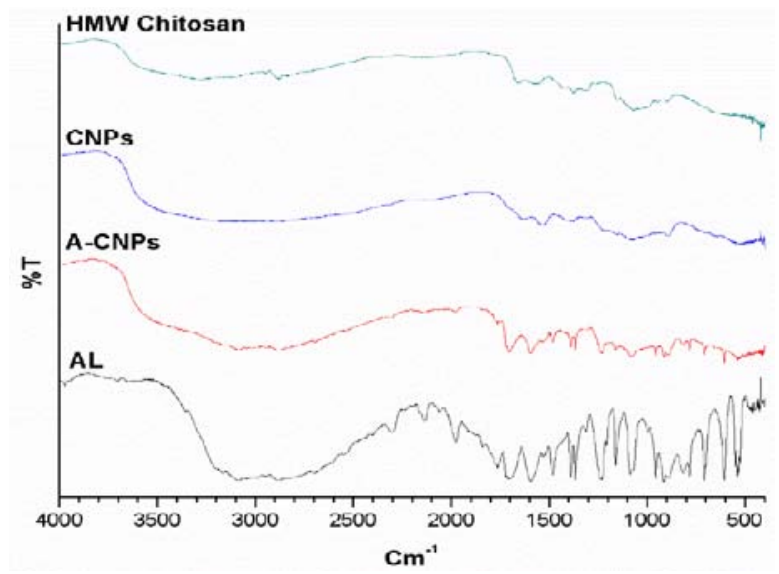

Fig. 3: FTIR spectra of pure drug AL, A-CNPs, CNPs, and HMW chitosan

\section{Differential scanning calorimetry}

DSC thermograms of AL, A-CNPs, CNPs, and P-AC have been shown in fig. 4. A sharp endothermic peak at $383^{\circ} \mathrm{C}$ was obtained in the DSC curve of $\mathrm{AL}$, demonstrating its crystallinity and melting point. There was no chemical interaction seen between pure AL and HMW chitosan in the DSC curve of P-AC, as the characteristic endothermic peak of AL $\left(383^{\circ} \mathrm{C}\right)$ and HMW chitosan $\left(101^{\circ} \mathrm{C}\right)$ appeared separately in the graph. In addition, the thermogram of P-AC showed an exothermic peak around $310{ }^{\circ} \mathrm{C}$ which corresponds to the degradation of amine unit of chitosan and this peak disappeared in the thermograms of CNPs and A-CNPs. Instead, a new prominent exothermic peak around $249^{\circ} \mathrm{C}$ was observed in thermogram of CNPs and A-CNPs. This is possibly due to the breakage of the electrostatic bond between polyanionic TPP and polycationic chitosan. Further, in the DSC thermogram of A-CNPs, no endothermic peak for $\mathrm{AL}$ was appeared around $383{ }^{\circ} \mathrm{C}$, which signifies that the $\mathrm{AL}$ drug had been incorporated into the chitosan matrix in an amorphous state. These results are further confirmed with XRD analysis [22].

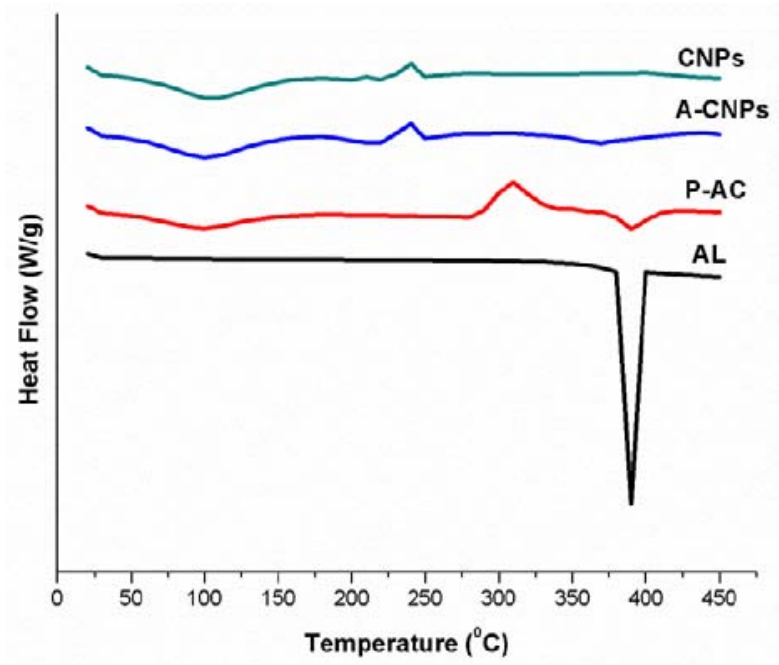

Fig. 4: DSC thermograms of pure drug AL, P-AC, A-CNPs and CNPs

\section{X-ray diffraction}

The physical state of AL and A-CNPs was examined by XRD analysis. The diffractograms of allopurinol and A-CNPs are shown in fig. 5 which demonstrates the high-intensity diffraction peaks for pure allopurinol drug $\left(2 \theta=14.61^{\circ}, 17.35^{\circ}, 25.80^{\circ}, 27.68^{\circ}\right)$, depicting its crystalline nature. However, the intensity of diffraction peaks of pure $\mathrm{AL}$ was reduced in A-CNPs formulation, confirming its successful encapsulation in the A-CNPs and its transformation from less soluble crystalline form to more soluble amorphous form [23].

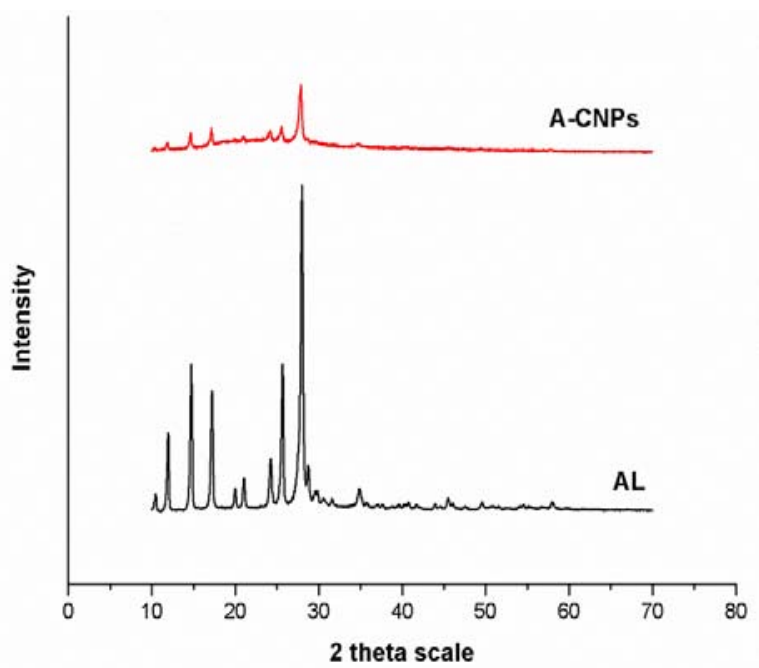

Fig. 5: XRD pattern of pure drug AL and A-CNPs

\section{CONCLUSION}

The present research work describes successful development of sustained release nanoparticlulate formulation of $\mathrm{AL}$ by its incorporation into chitosan-TPP matrix. The particle size of prepared A-CNPs lied in the nano range and they showed a sustained release pattern. Polydispersity index and ZP values depicts the formulation to be stable. DSC and XRD studies affirms the transformation of physical state of AL in formulation to amorphous state (more soluble form). Further, in vivo studies can improve their potential role in drug targeting and for the benefit of mankind.

\section{ACKNOWLEDGMENT}

Authors are extremely thankful to Late Dr. Shailendra Kumar Singh (Professor), Department of Pharmaceutical Sciences, GJUSandT, Hisar for guiding throughout the research work. Authors express thankfulness to the University Grants Commission, New Delhi, for providing Rajiv Gandhi National Fellowship. The authors are thankful to Coordinator, DST-FIST, Department of Pharmaceutical Sciences, GJUSandT, Hisar for providing particle size analysis.

\section{AUTHORS CONTRIBUTIONS}

All the author have contributed equally

\section{CONFLICTS OF INTERESTS}

\section{Declared none}

\section{REFERENCES}

1. Anderson EE, Rundles RW, Silberman HR, Metz EN. Allopurinol control of hyperuricosuria: a new concept in the prevention of uric acid stones. J Urol 1967;97:344-7.

2. Ngo TC, Assimos DG. Uric acid nephrolithiasis: recent progress and future directions. Rev Urol 2007;9:17.

3. De Vries A, Frank M, Liberman UA, Sperling O. Allopurinol in the prophylaxis of uric acid stones. Ann Rheum Dis 1966;25:691.

4. Younis MK, Tareq AZ, Kamal IM. Optimization of swelling, drug loading and release from natural polymer hydrogels. IOP Conf Ser: Mater Sci Eng 2018;454. Doi:10.1088/1757-899X/454/ $1 / 012017$.

5. Mohanraj VJ, Chen Y. Nanoparticles-a review. Trop J Pharm Res 2006;5:561-73. 
6. Nikam AP, Mukesh PR, Chaudhary SP. Nanoparticles-an overview. Int J Res Dev Pharm Life Sci 2014;3:1121-7.

7. Priya J, Naha A, Dhoot AS, Xalxo N. A review on polymeric nanoparticles: A promising novel drug delivery system. J Global Pharma Technol 2018;10:10-7.

8. Agnihotri SA, Mallikarjuna NN, Aminabhavi TM. Recent advances on chitosan-based micro-and nanoparticles in drug delivery. J Controlled Release 2004;100:5-28.

9. Nagpal K, Singh SK, Mishra DN. Formulation, optimization, in vivo pharmacokinetic, behavioral and biochemical estimations of minocycline loaded chitosan nanoparticles for enhanced brain uptake. Chem Pharm Bull 2013;61:258-72.

10. Patel BK, Parikh RH, Aboti PS. Development of oral sustained release rifampicin loaded chitosan nanoparticles by design of an experiment. J Drug Delivery 2013. Doi:10.1155/2013/ 370938.

11. Kandav G, Singh SK. Review of nanoemulsion formulation and characterization techniques. Indian J Pharm Sci 2018;80:781-9.

12. Manimekalai P, Dhanalakshmi R, Manavalan R. Preparation and characterization of ceftiaxone sodium encapsulated chitosan nanoparticles. Int J Appl Pharm 2017;9:10-5.

13. Girotra P, Thakur A, Kumar A, Singh SK. Identification of multitargeted anti-migraine potential of nystatin and development of its brain targeted chitosan nanoformulation. Int J Biol Macromol 2017;96:687-96.

14. Hussain Z, Sahudin S. Preparation, characterisation and colloidal stability of chitosan-tripolyphosphate nanoparticles: optimisation of formulation and process parameters. Int J Pharm Pharm Sci 2016;8:297-308.
15. Hansraj GP, Singh SK, Kumar P. Sumatriptan succinate loaded chitosan solid lipid nanoparticles for enhanced anti-migraine potential. Int J Biol Macromol 2015;81:467-76.

16. Sreelola VU, Sailaja AK, Pharmacy M. Preparation and characterisation of ibuprofen-loaded polymeric nanoparticles by a solvent evaporation technique. Int J Pharm Pharm Sci 2014;6:416-21

17. Girotra P, Singh SK. A comparative study of orally delivered PBCA and ApoE coupled BSA nanoparticles for brain targeting of sumatriptan succinate in the therapeutic management of migraine. Pharm Res 2016;33:1682-95.

18. Tayal K, Kandav G, Girotra P, Singh SK. Formulation and evaluation of chitosan-coated magnetic nanoparticles of Amoxicillin trihydrate. Pharm Lett 2015; 7:241-51.

19. Shende P, Yadava SK, Patil PS. Development and characterization of chitosan nanoparticles containing erthromycin estolate. Int J Pharm Appl 2014;5:1-7.

20. Changdeo JS, Vinod M, Shankar KB, Rajaram CA. Physicochemical characterization and solubility enhancement studies of allopurinol solid dispersions. Braz J Pharm Sci 2011;47:513-23.

21. Gierszewska Drużynska M, Ostrowska Czubenko J. The effect of ionic crosslinking on thermal properties of hydrogel chitosan membranes. Prog Chem Appl Chitin Its Deriv; 2010. p. 25-32.

22. Hanafy AS, Farid RM, ElGamal SS. Complexation as an approach to entrap cationic drugs into cationic nanoparticles administered intranasally for Alzheimer's disease management: preparation and detection in rat brain. Drug Dev Ind Pharm 2015;41:2055-68.

23. Shelake SS, Patil SV, PATIL SS, Sangave P. Formulation and evaluation of fenofibrate-loaded nanoparticles by precipitation method. Indian J Pharm Sci 2018;80:420-7. 